\title{
Alexithymia in eating disorders: therapeutic implications
}

This article was published in the following Dove Press journal:

Psychology Research and Behavior Management

22 December 2014

Number of times this article has been viewed

\section{Federica Pinna \\ Lucia Sanna \\ Bernardo Carpiniello \\ Department of Public Health, Clinical and Molecular Medicine - Unit of Psychiatry, University of Cagliari, Cagliari, Italy}

\begin{abstract}
A high percentage of individuals affected by eating disorders (ED) achieve incomplete recovery following treatment. In an attempt to improve treatment outcome, it is crucial that predictors of outcome are identified, and personalized care approaches established in line with new treatment targets, thus facilitating patient access to evidence-based treatments. Among the psychological factors proposed as predictors of outcome in ED, alexithymia is of outstanding interest. The objective of this paper is to undertake a systematic review of the literature relating to alexithymia, specifically in terms of the implications for treatment of ED. In particular, issues concerning the role of alexithymia as a predictor of outcome and as a factor to be taken into account in the choice of treatment will be addressed. The effect of treatments on alexithymia will also be considered. A search of all relevant literature published in English using PubMed, PsycINFO, and Scopus databases was carried out on the basis of the following keywords: alexithymia, anorexia nervosa, bulimia nervosa, eating disorders, and treatment; no time limits were imposed. Despite the clinical relevance of alexithymia, the number of studies published on the above cited aspects is somewhat limited, and these studies are largely heterogeneous and feature significant methodological weaknesses. Overall, data currently available mostly correlate higher levels of alexithymia with a less favorable outcome in ED. Accordingly, alexithymia is seen as a relevant treatment target with the aim of achieving recovery of these patients. Treatments focusing on improving alexithymic traits, and specifically those targeting emotions, seem to show greater efficacy, although alexithymia levels often remain high even after specific treatment. Further investigations are needed to overcome the methodological limitations of previous studies, to understand the actual impact of alexithymia on ED outcome, and to allow more precise implications for treatment to be drawn. Additional research should also be undertaken to specify which of the alexithymic dimensions are specifically relevant to the course and outcome of ED, and to identify treatment protocols producing a significantly greater efficacy in ED patients with relevant alexithymic traits.
\end{abstract}

Keywords: anorexia nervosa, bulimia nervosa, treatment

\section{Introduction}

A high percentage of individuals with eating disorders (ED) achieve incomplete recovery following treatment. ${ }^{1}$ In an attempt to increase the efficacy of ED treatments, the identification of predictors of outcome, adoption of individually tailored interventions based on new treatment targets, and facilitation of access of ED patients to evidencebased treatments ${ }^{2}$ should be addressed as a matter of urgency. Cognitive behavioral therapy (CBT), antidepressants, and interpersonal therapy (IPT) have been suggested as evidence-based treatments in bulimia nervosa $(\mathrm{BN})$ and binge eating disorder (BED). ${ }^{3}$ Limited benefits have been reported in the pharmacotherapy of anorexia nervosa (AN),
Correspondence: Bernardo Carpiniello Department of Public Health, Clinical and Molecular Medicine - Unit of Psychiatry, University of Cagliari, Via Liguria I3, 09127 Cagliari, Italy

Email bcarpini@iol.it submit your manuscript | www.dovepress.com

Dovepress

http://dx.doi.org// 0.2147/PRBM.S52656
Psychology Research and Behavior Management 2015:8 I-I5

(c) (1) (5) 2015 Pinna et al. This work is published by Dove Medical Press Limited, and licensed under Creative Commons Attribution - Non Commercial (unported, v3.0) License. The full terms of the License are available at http://creativecommons.org/licenses/by-nc/3.0/. Non-commercial uses of the work are permitted without any further permission from Dove Medical Press Limited, provided the work is properly attributed. Permissions beyond the scope of the License are administered by Dove Medical Press Limited. Information on how to request permission may be found at: http://www.dovepress.com/permissions.php 
and no single psychological intervention has demonstrated a clear superiority in adults with AN, both in terms of effectiveness and adherence to treatment. ${ }^{4}$ However, recently emerging data on the effectiveness of family therapy in adolescents with AN have been reported. ${ }^{4}$ Although effective, and displaying better results than in $\mathrm{AN}$, treatments for $\mathrm{BN}$ are characterized by optimal responses in no more than $50 \%$ of cases. ${ }^{1}$ Among psychological factors proposed as predictors of outcome in the ED, alexithymia has elicited a growing interest. Coined by Sifneos, ${ }^{5}$ the term alexithymia was initially introduced to describe a cognitive style and affective characteristic of psychosomatic patients, consisting in difficulty in identifying and communicating emotions and in discriminating them from bodily sensations, with a limited fantasy and a concrete, externally oriented cognitive style. Subsequently, the construct evolved, expanding beyond the area of psychosomatic diseases, and has been implicated in a series of physical and mental disorders, including ED. ${ }^{6}$ Development of the self-administered Toronto Alexithymia Scale (TAS), ${ }^{7}$ and, in particular, of its 20 -item form (TAS-20), ${ }^{8}$ has contributed largely to the dissemination of studies on alexithymia, providing a reliable and valid measure of the construct, although a multimeasure multimethod has been suggested in order to improve the quality of research. ${ }^{6}$ In general, high levels of alexithymia have been found in subjects with ED, with specific deficits in identifying and communicating emotions. ${ }^{9}$ While many studies have reported no significant differences according to diagnostic subgroups of ED, other studies have shown higher levels of alexithymia in AN, particularly with regard to the difficulty in communicating emotions. ${ }^{9}$ Levels of alexithymia are generally reported to improve following treatment of ED, although the clinical relevance of this change is questionable, taking into account that, in the majority of cases, alexithymia levels remain elevated even after treatment. ${ }^{9}$ Whether alexithymia should be considered a state-dependent variable or a trait remains open to debate. Research findings tend to support the hypothesis whereby alexithymia cannot simply be considered as depending on ED symptoms alone, as the role of anxiety and depression on alexithymia remains controversial, and the presence of conflicting data underline the need for further investigations using a more appropriate methodology. ${ }^{9}$ Moreover, the characterization of alexithymia as a stable personality trait or state-dependent phenomenon is often discussed in terms of absolute and relative stability; ie, levels of alexithymia may vary to reflect a change in symptom severity (lack of absolute stability), while the relative differences of scores between patients remain the same (relative stability of the construct). ${ }^{6}$ Prevalent evidence supporting the relative stability of the construct has led researchers to investigate the possible predictive power of alexithymia on treatment outcome. Based on these premises, the aim of this paper was to summarize and discuss data present in literature regarding: 1) the implications of alexithymia as a predictor of outcome in ED treatment, and as a variable influencing choice of treatments; and 2) the effect of treatments on alexithymia levels in ED patients.

\section{Methods}

This paper provides a systematic review of the literature on the implications of alexithymia on treatment, and the effect of treatments on alexithymia levels, in individuals with ED (AN, BN, BED, and/or ED not otherwise specified [EDNOS]). A literature search with no time limits was carried out in July 2014 on PubMed, PsycINFO, and Scopus databases. Only papers written in English were considered for the purposes of this review. The search was conducted using the keywords alexithymia, anorexia nervosa, bulimia nervosa, eating disorders, and treatment. The initial search resulted in the identification of 828 abstracts, which fell to 357 after the elimination of duplicates. Abstracts were then reviewed and studies selected on the basis of the following criteria: 1) measurement of alexithymia using standardized instruments, namely one of the three versions of the TAS; 2) reporting of data on the effects of specific treatments on alexithymia in individuals with ED; 3) reporting of data on the impact of alexithymia on treatment outcomes in individuals with ED; and 4) reporting of data on the impact of alexithymia on choice of treatment in individuals with ED. Twenty-seven articles were identified. Following further examination of the full text, 15 papers were finally selected and considered for the present review. Twelve articles did not meet the inclusion criteria as described.

\section{Results}

The 15 selected studies are summarized in Tables 1 and 2 . Ten studies focused on the implications of alexithymia as a predictor of treatment outcome in subjects affected by ED, one study analyzed the implications of alexithymia on treatment choices for individuals with ED, and ten studies investigated the effects of specific treatments on levels of alexithymia in individuals with ED.

\section{Alexithymia as a predictor of treatment outcome in patients with ED Overview of studies}

Ten of 15 studies selected for this review focused on alexithymia as a predictor of treatment outcome in patients 


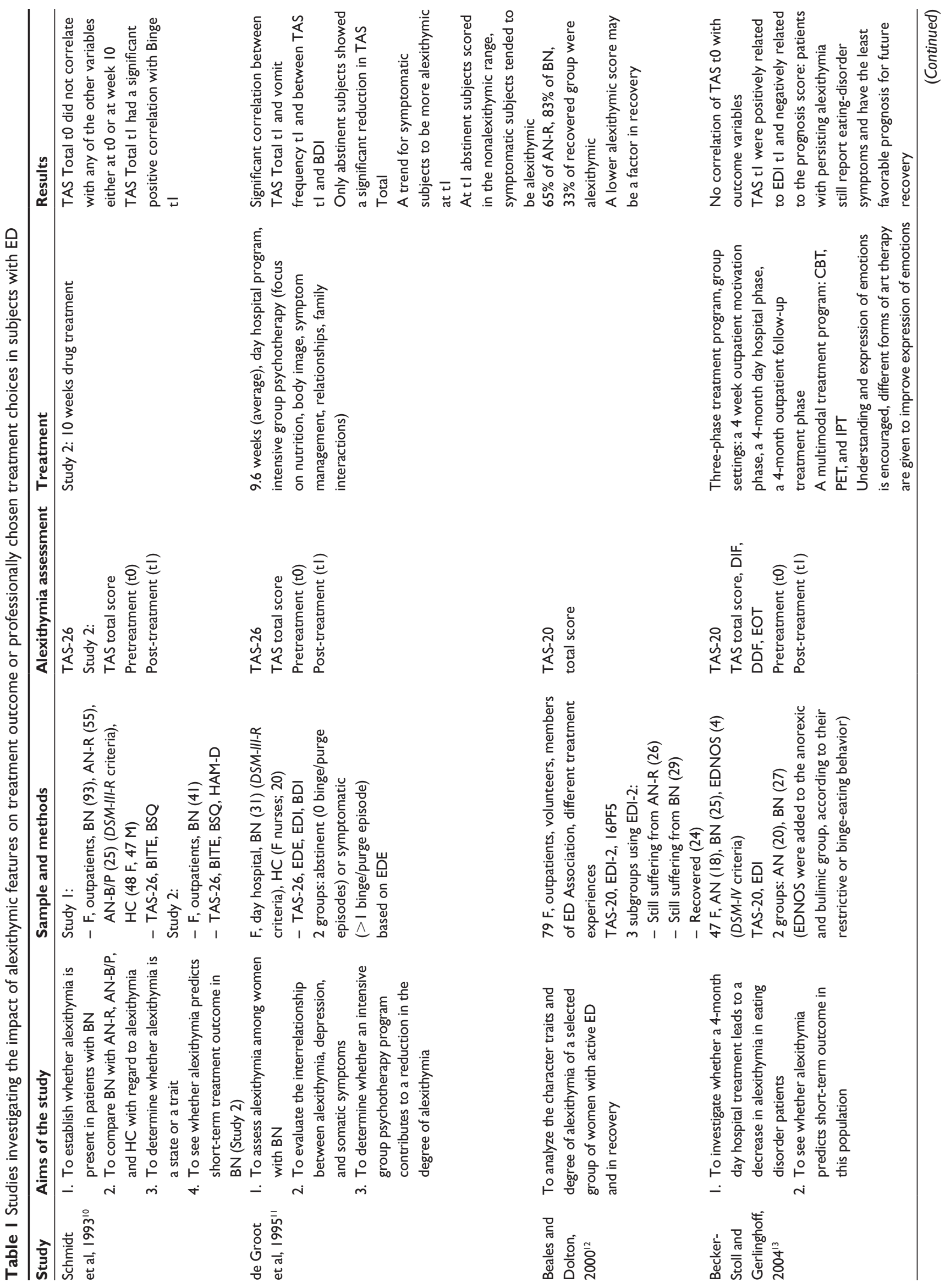




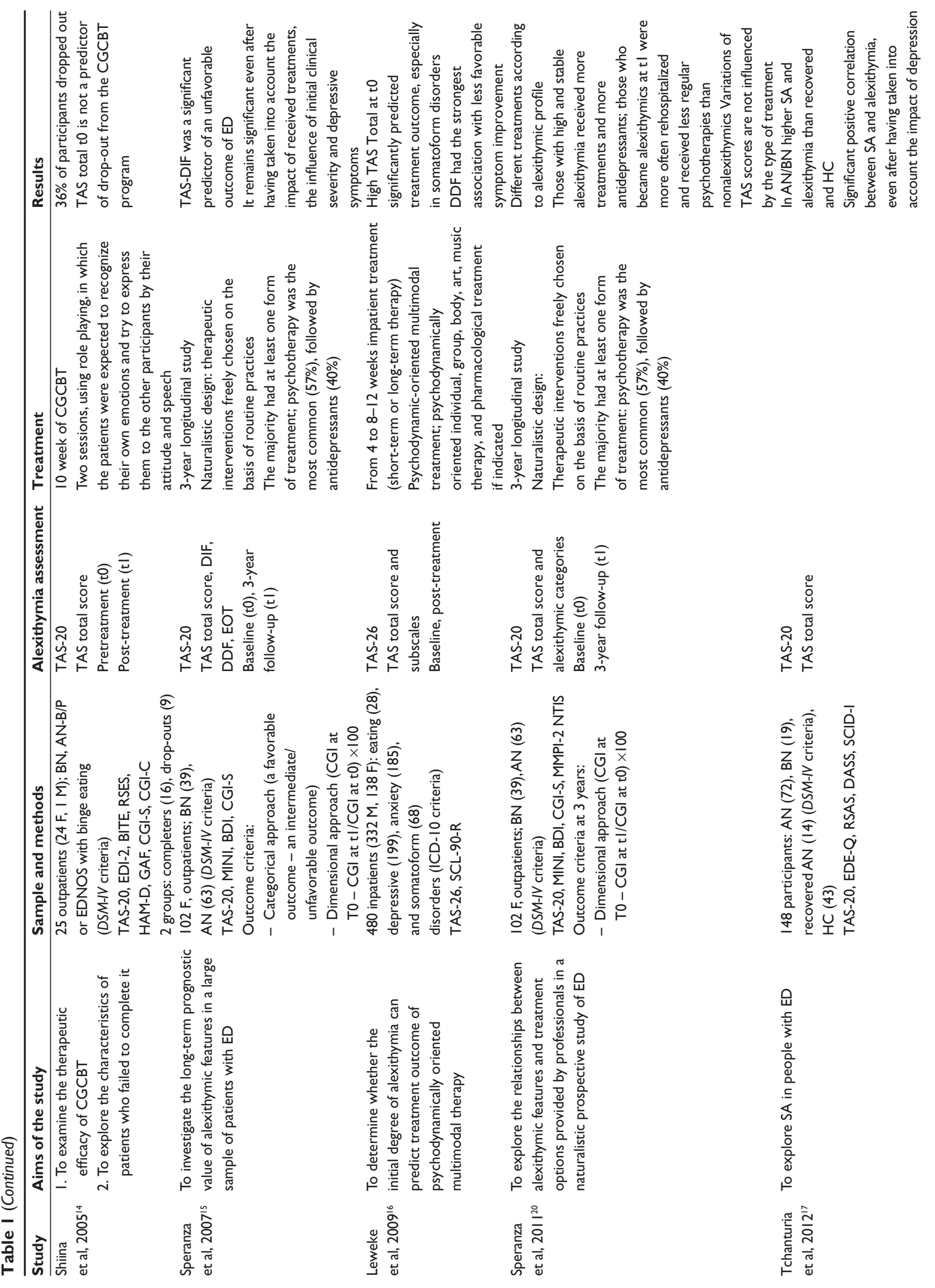




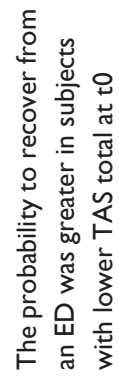

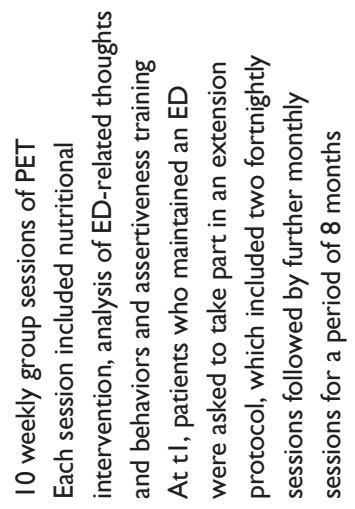

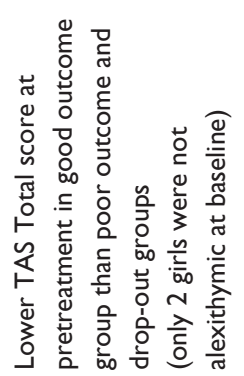
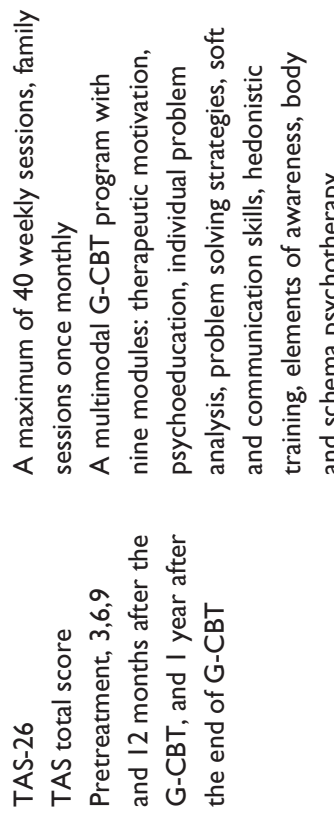
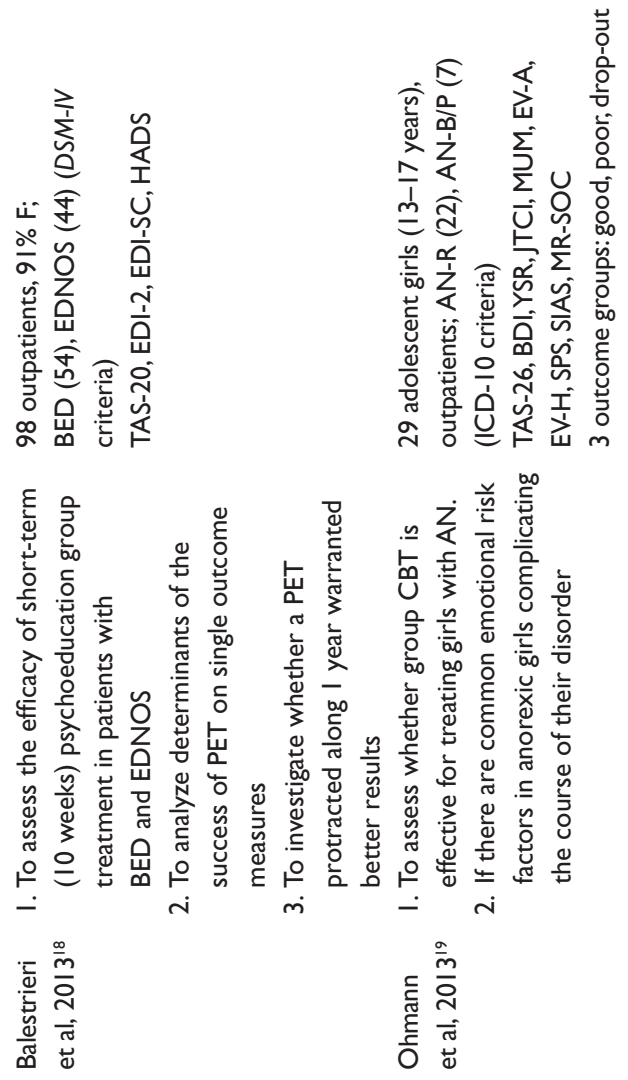

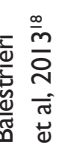

造)

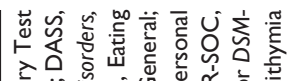

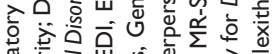

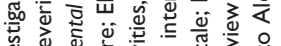

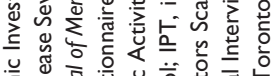

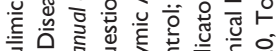

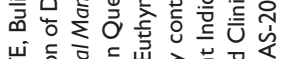

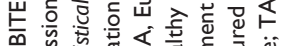

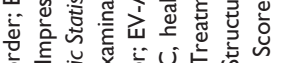

等

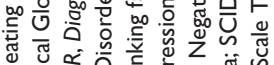

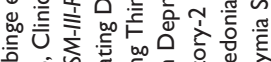

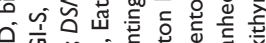

㟧 ज寸

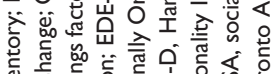

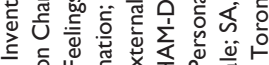

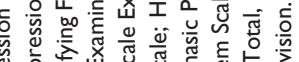

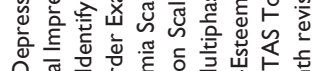

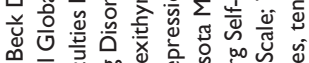

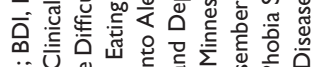

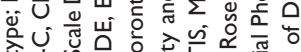

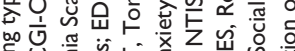

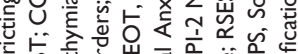

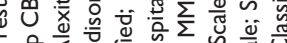

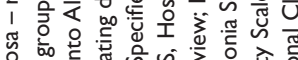

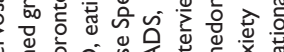

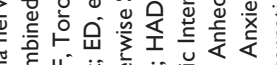

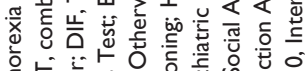

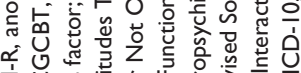

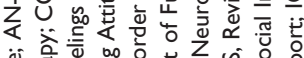

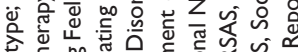

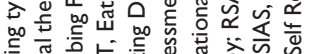

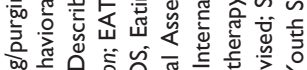

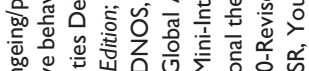

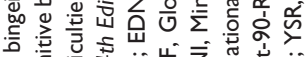

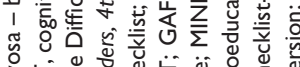

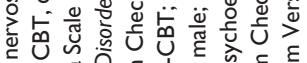

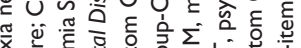

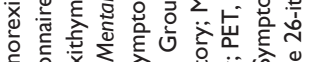

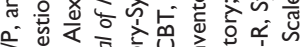

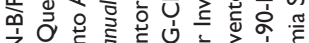

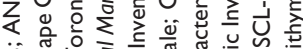

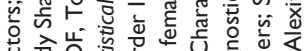

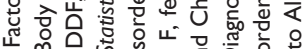

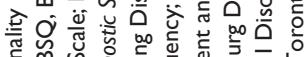

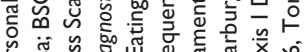

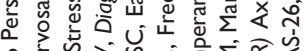

일

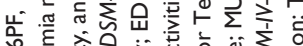

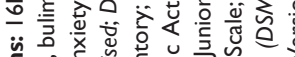

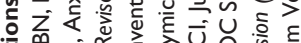

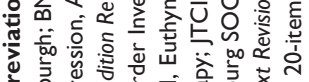

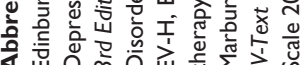




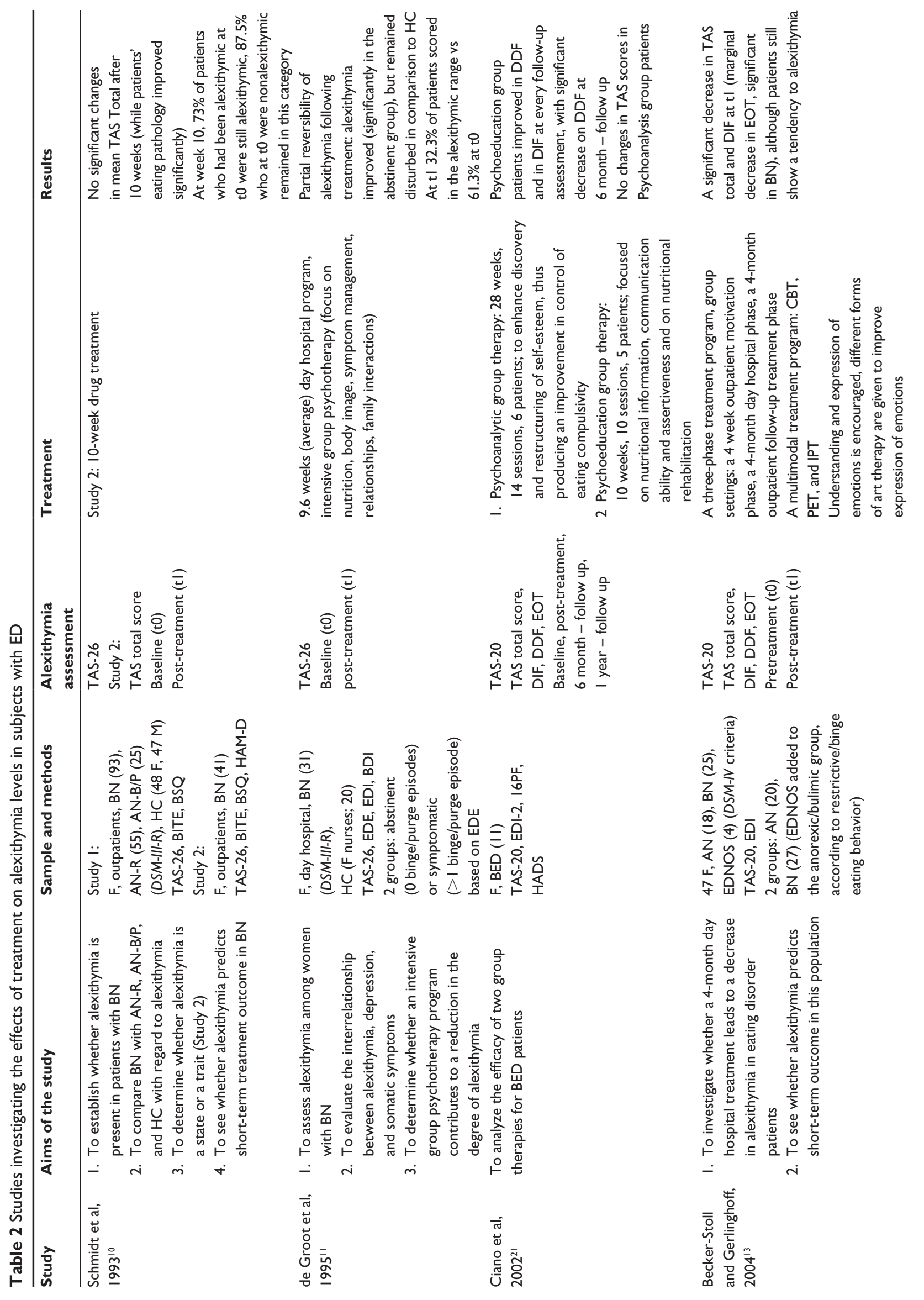



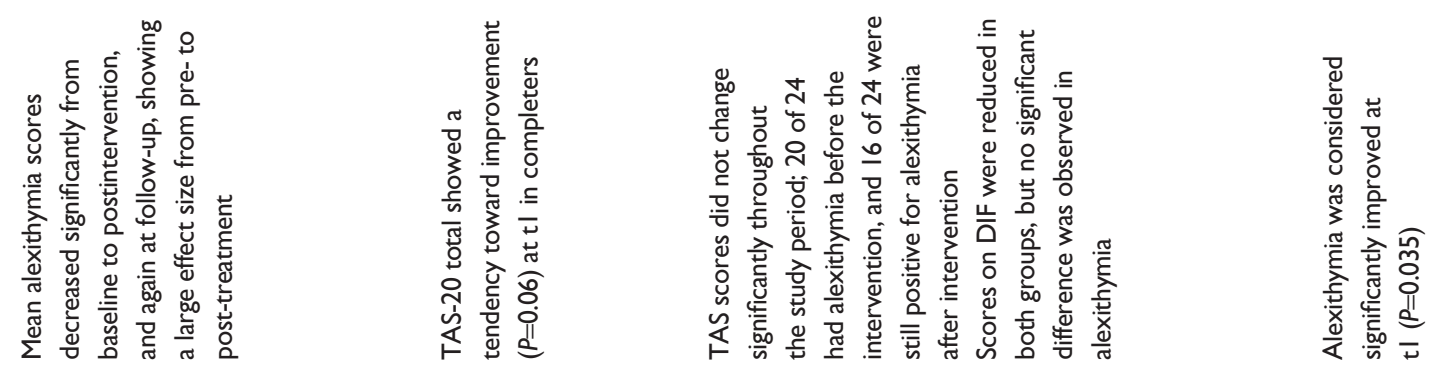

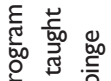

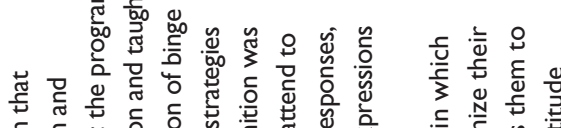

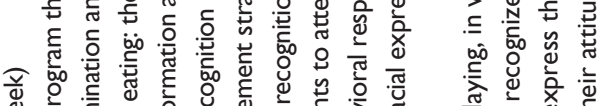

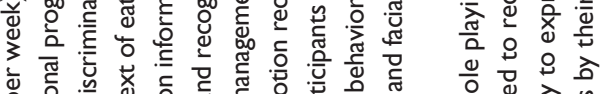

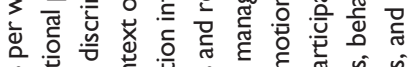

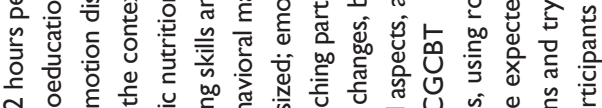

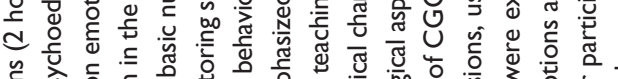

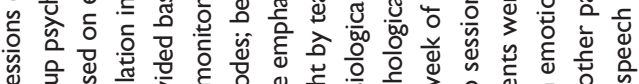

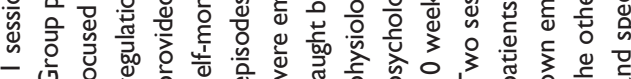

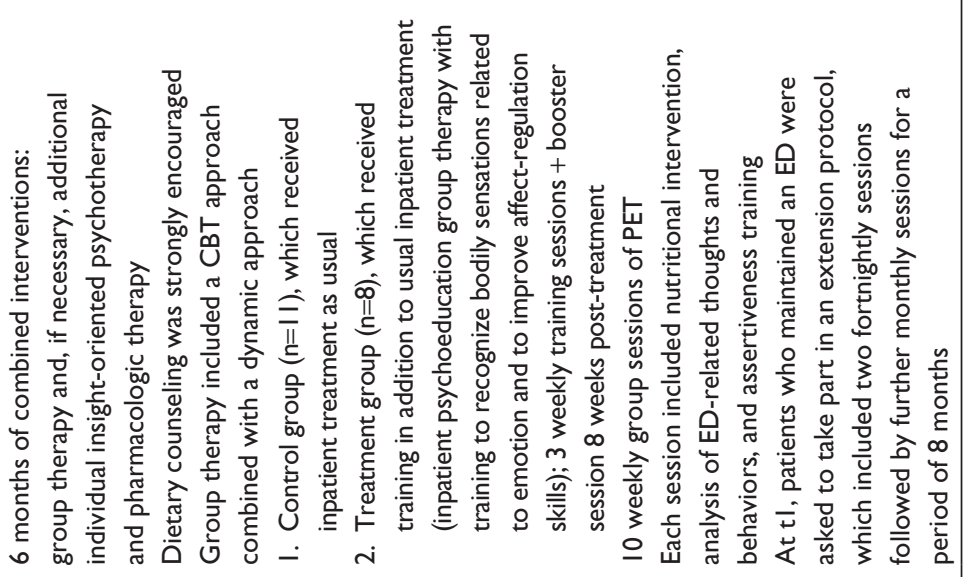

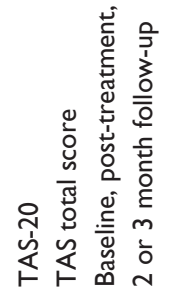
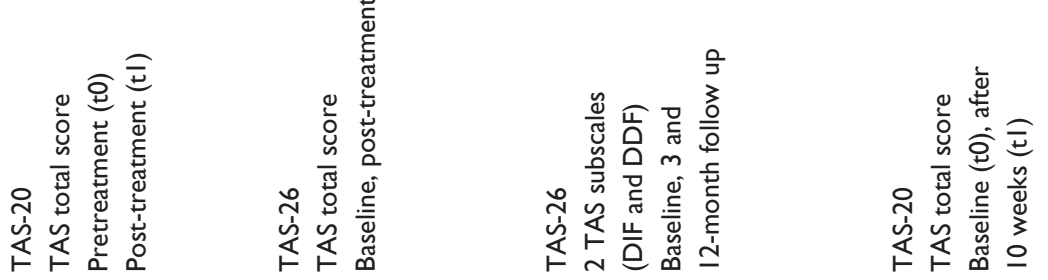

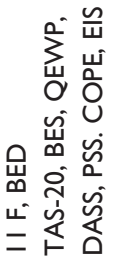

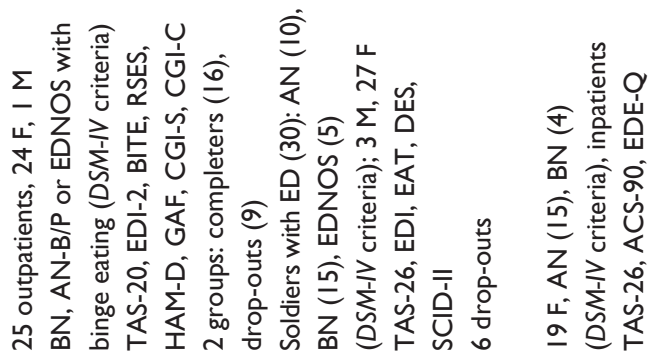

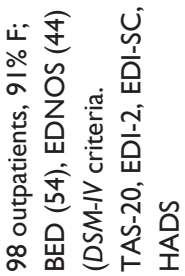
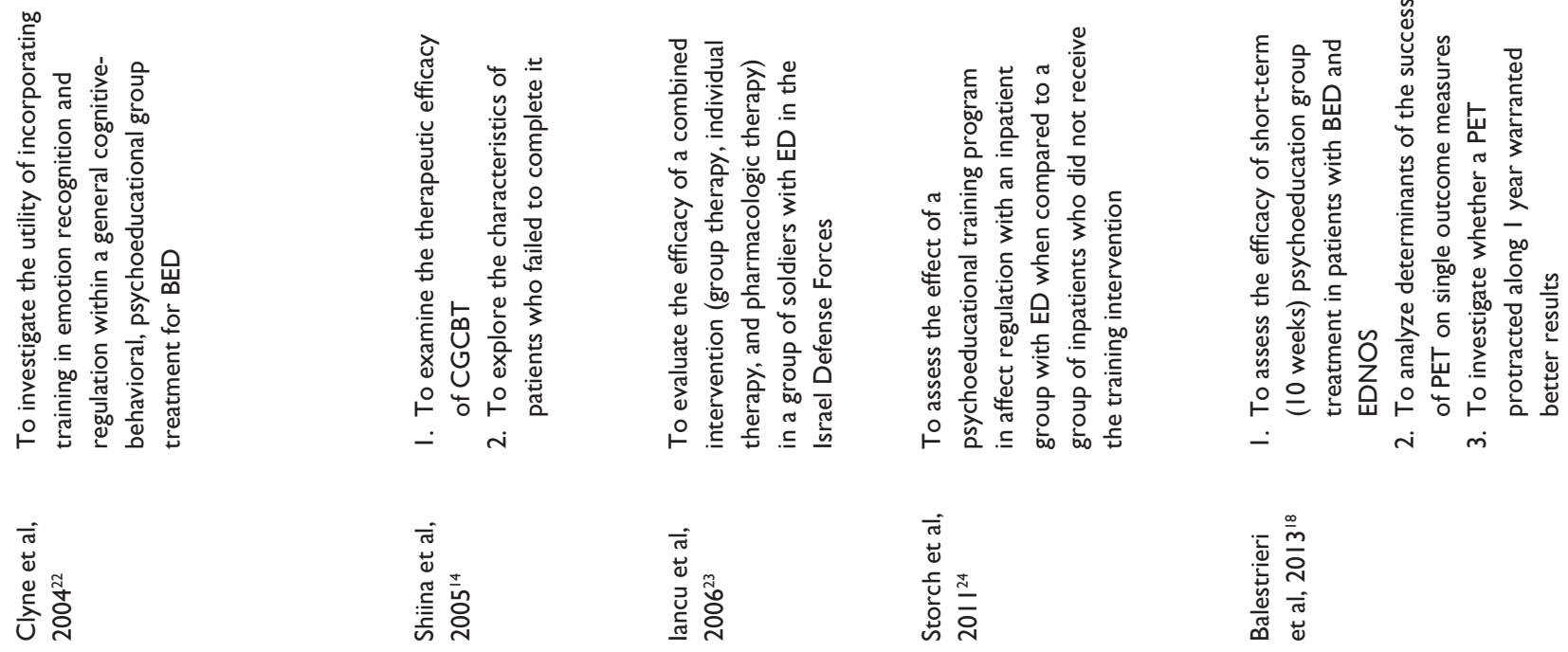

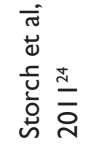

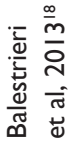




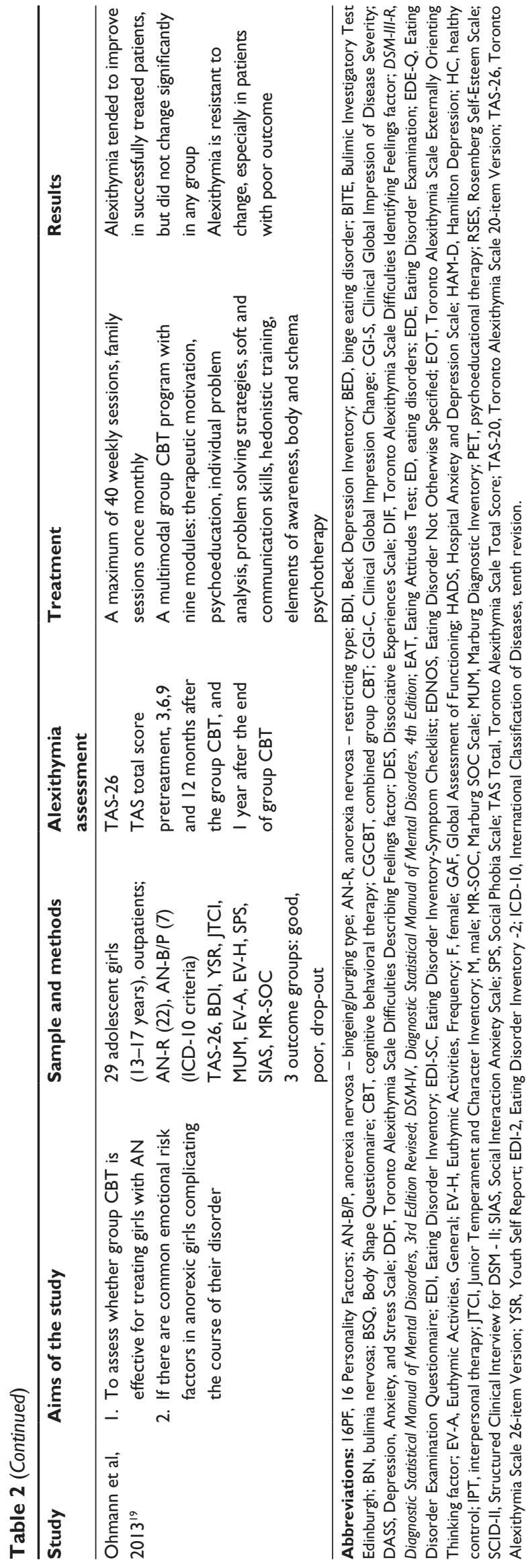

with ED. ${ }^{10-19}$ Of these, eight were based on a longitudinal design, ${ }^{10,11,13-16,18,19}$ while two used a cross-sectional design. ${ }^{12,17}$ Methodology and treatment protocol adopted by each study are shown in Table 1. All studies were conducted on samples of exclusively, or predominantly, female patients. Three studies included a control group of subjects without ED. ${ }^{10,11,17}$ The diagnostic groups analyzed are, in the majority of cases, mixed groups of AN, BN, and possibly EDNOS. In two cases the sample was made up exclusively of bulimic patients, ${ }^{10,11}$ while one study considered both patients with BED and EDNOS. ${ }^{18}$ Three studies took into account not only TAS total score, but also scores of its dimensions. ${ }^{13,15,16}$ In the context of the longitudinal studies, those conducted by Speranza et al, ${ }^{15}$ Leweke et al, ${ }^{16}$ and Balestrieri et al ${ }^{18}$ showed a significant predictive power of baseline alexithymia with respect to treatment outcome; indeed, a high TAS total score generally predicted a worse outcome, and among TAS dimensions, Difficulties Identifying Feelings (DIF) ${ }^{15}$ and Difficulties Describing Feelings (DDF $)^{16}$ displayed a greater predictive power. Other studies have demonstrated a significant correlation between alexithymia and outcome variables only at post-treatment evaluation, with higher levels of alexithymia emerging in more symptomatic patients. ${ }^{10,11,13}$ Only one of the longitudinal studies reported negative results (no correlation between alexithymia and outcome variables). ${ }^{14}$ The two cross-sectional studies demonstrated, respectively, lower rates of alexithymia among recovered ED patients ${ }^{12}$ and higher levels of alexithymia among ED patients who were still symptomatic both compared to recovered patients and to healthy controls. ${ }^{17}$

\section{Analysis of single studies Longitudinal studies}

The study by Schmidt et al ${ }^{10}$ was the first to analyze alexithymia as a predictor of outcome of short-term treatment of BN adopting a prospective design;. The study recruited 41 female patients affected by BN according to Diagnostic and Statistical Manual of Mental Disorders, 3rd Edition Revised (DSM-III-R) who were submitted to a 10-week treatment program; the treatment plan included both psychotherapy and drug treatment (fluvoxamine). TAS-26 total score at baseline was not related to any outcome variable considered; however, TAS total score at 10 weeks was positively correlated to the number of binges. Given that the majority of patients were not recovered at the end of treatment, the authors hypothesized that persistence of eating symptoms could be related to persistence of high levels of alexithymia. 
Two years later, de Groot et $\mathrm{al}^{11}$ evaluated alexithymia in a sample of 31 women with severe BN according to DSM-III-R. All patients were admitted to the Toronto Hospital Day Hospital Program for ED, which included an intensive 9.6-week group psychotherapy. After treatment, patients were divided into two groups defined as abstinent (no binge/purge episodes) or symptomatic ( $>1$ binge/purge episode), based on the frequency of relevant symptoms during the 28-day period prior to discharge. Following treatment, differences in emotional awareness between abstinent and symptomatic groups were found to be clinically relevant; indeed, nonalexithymic subjects were detected predominantly in the abstinent group, while alexithymics were mostly found among symptomatic patients. At discharge, abstinence from binge eating and vomiting episodes in the last 4 weeks was associated with a significant reduction of TAS-26 total scores; moreover, a positive correlation was observed between TAS scores, level of depression, and frequency of episodes of vomiting. At the end of treatment, therefore, patients with higher alexithymic scores were more symptomatic in terms of ED and depression symptoms.

Becker-Stoll and Gerlinghoff ${ }^{13}$ evaluated 47 female patients with $\mathrm{ED}$ (18 with $\mathrm{AN}, 25$ with $\mathrm{BN}$, and four with EDNOS according to the DSM Fourth Edition [DSM-IV]) as part of a 4-month outpatient group treatment program at the Treatment Center for Eating Disorders of the Max Planck Institute of Psychiatry in Munich. The center offered a three-phase treatment program: an outpatient motivation phase (4 weeks), a day hospital treatment phase (4 months), and a 4-month follow-up phase. Treatment methods included CBT, psychoeducational therapy (PET), and IPT. Patients were divided into two groups (patients affected by anorexia and bulimia). The study revealed no correlation between TAS total score at baseline and outcome variables. However, TAS total scores after treatment correlated positively with Eating Disorder Inventory (EDI) scores, and negatively with a specific prognostic score; indeed, patients with persistent alexithymia were characterized by a higher number of ED symptoms and a less favorable prognosis.

In a subsequent open-label study of 25 patients ( 24 females, one male) with bulimic symptoms (BN, AN-B/P, EDNOS, and BED), Shiina et $\mathrm{al}^{14}$ examined predictors of drop-outs from treatment programs; subjects underwent an outpatient 10-week group therapy, combining CBT with assertiveness training and self-esteem reinforcement therapy (CBT combined group). Thirty-six percent of participants failed to complete the program, but alexithymia was not predictive of drop-out.

Speranza et $a 1^{15}$ investigated the prognostic value of alexithymia in a sample of ED patients in the context of a 3-year prospective, naturalistic follow-up study. This study represents the first longitudinal project to investigate the predictive value of alexithymia respect to long-term clinical outcome of patients with ED. The sample consisted of 102 young women, with an average or high level of education and a severe, chronic ED, including $63 \mathrm{AN}$ and $39 \mathrm{BN}$ patients, diagnosed according to $D S M-I V$ criteria. The study featured a naturalistic design, with therapeutic interventions being freely chosen in line with routine clinical practice. Patients with a comorbid major depressive episode or alcohol or drug addiction were excluded in order to obtain a more homogeneous sample, by excluding confounding factors. Clinical outcome at 3 years was defined both in terms of categorical diagnosis (presence or absence of criteria for diagnosis of ED) and in terms of clinical improvement between baseline and follow-up. Based on the presence of ED symptoms, two categories of outcome were defined: favorable, characterized by a complete disappearance of ED symptoms at follow-up, and intermediate/unfavorable, characterized by persistence of a subsyndromic condition or full ED syndrome. Overall, a negative outcome emerged, with $75 \%$ of patients featuring an intermediate/unfavorable outcome, and only $25 \%$ a favorable outcome. The majority of patients underwent at least one type of therapeutic intervention over the 3-year period, mostly psychotherapy (57\%), followed by the prescription of drugs, generally antidepressants (40\%). DIF dimension of TAS-20 revealed to be a significant predictor of unfavorable outcome, being negatively correlated to clinical improvement. The predictive power of TAS-DIF dimension alone emerged as significant, albeit to a lower degree, in a second predictive model that took into account the impact of depressive symptomatology (evaluated by means of BDI), clinical severity (evaluated through CGI), and treatments received. Indeed, patients showing more difficulty identifying emotions at baseline were found to be more often symptomatic at followup, with a less favorable clinical course.

Leweke et $\mathrm{al}^{16}$ studied 28 patients with ED, diagnosed according to International Classification of Diseases, 10th revision (ICD-10) criteria, in the context of a large study of 480 patients affected by a series of different diagnostic conditions (ED, depressive disorder, anxiety disorder, acute or post-traumatic stress disorder, and somatoform disorder) who underwent multimodal psychodynamic intervention on an inpatient basis. In particular, treatment program included both individual and group psychodynamically oriented therapy, art and music therapy, and pharmacological treatment, if indicated. Duration of treatments ranged from 4 weeks to 8-12 weeks. A high basal level of alexithymia evaluated by 
means of TAS-26 was in general a significant predictor of treatment outcome, but considering each single diagnostic group, significance was maintained only for patients with somatoform disorder, with the strongest predictive power found for the DDF dimension: patients obtaining higher scores displayed a less favorable course and outcome of the disorder.

Balestrieri et al ${ }^{18}$ analyzed determinants of success of short-term (10 weeks) group psychoeducation delivered on an outpatient basis to 98 patients ( $91 \%$ women) with BED $(\mathrm{n}=54)$ and EDNOS $(\mathrm{n}=44)$, according to DSM- $I V$ criteria. The probability of being in recovery at the endpoint was higher for subjects with low alexithymia scores at baseline TAS-20.

The study by Ohmann et $\mathrm{al}^{19}$ examined the emotional assets of 29 adolescent girls with AN who underwent a 10-month group CBT, carried out on an outpatient basis. On request, individual CBT intervention was offered. Evaluations were performed at baseline, during treatment (at 3,6, and 9 months), 1 year after the start of the study, and 1 year after the end of treatment. Patients were divided into three groups on the basis of outcome: $\operatorname{good}(n=16)$, poor $(n=5)$, and drop-outs $(n=8)$. At all time points, slightly lower scores on the TAS were detected in the good outcome compared to the poor outcome and the drop-out group, with a tendency toward lower alexithymia scores in successfully treated patients. The drop-out patients were those with the highest baseline TAS-26 scores.

\section{Cross-sectional studies}

The study by Beales and Dolton ${ }^{12}$ evaluated 79 women affected by chronic, severe ED treated with an array of therapeutic approaches. Patients were recruited by means of a letter sent to 200 members of the Eating Disorders Association who were invited to take part in the study. Those who joined the study were divided into three groups (anorexic, bulimic, and recovered) on the basis of EDI - 2 scores. Based on total score obtained at TAS-20, 65\% of patients affected by anorexia, $83 \%$ of those affected by nervous bulimia, and $33 \%$ of recovered ED patients were found to be alexithymic, with a significant difference between those who were still symptomatic and those who were recovered. In line with these results, the authors speculate that lower levels or absence of alexithymia may favor recovery of patients with ED. However, the study was not designed to determine whether recovered subjects were alexithymic or not before treatment or to detect any changes in alexithymia levels following treatment of ED.

The study of Tchanturia et a ${ }^{17}$ was based on the assumption that social anhedonia (a reduced sense of pleasure from social stimulation and reduced interest to look for social interaction) may represent an important symptom dimension in ED. The study evaluated levels of social anhedonia and its correlation with alexithymia in ED patients, both symptomatic and recovered, compared to healthy controls. Of 148 subjects recruited, 91 were affected by an ED diagnosed according to DSM-IV. Participants were divided into four groups: outpatient and hospitalized AN patients $(n=72)$, outpatient $\mathrm{BN}$ patients $(\mathrm{n}=19)$, AN patients who were recovered $(n=14)$, and healthy controls with no family or personal history of mental illness $(n=43)$. Symptomatic ED patients showed higher levels of both social anhedonia and alexithymia compared to recovered subjects, who showed an intermediate level of TAS total score, and to healthy controls, who displayed the lowest levels of alexithymia at TAS-20. Higher levels of social anhedonia correlated both with higher severity of ED symptoms and higher levels of alexithymia, even after controlling for severity of depression.

\section{Alexithymia as a predictor of treatment choice for ED patients}

The only study to investigate the role of alexithymia as a predictor of treatment choice for ED patients was published by Speranza et al. ${ }^{20}$ The authors analyzed the relationship between alexithymic characteristics and treatment options for ED adopted by clinicians as part of a prospective, 3-year naturalistic follow-up study of a sample of 102 patients with a diagnosis of ED. Indeed, the results of the study demonstrated that patients received different treatments, both qualitatively and quantitatively, in relation to their alexithymia profile. Patients with high and stable levels of alexithymia were characterized by a tendency to receive, on the whole, the most treatments of any kind and, significantly, more antidepressants than nonalexithymic patients, despite similar levels of depression at the time of inclusion in the study. Patients who were not alexithymic at baseline but developed alexithymic traits during the follow-up period were more often hospitalized and received psychotherapy less regularly than constantly nonalexithymic subjects. Changes over time in scores obtained at TAS-20 were not influenced by the use of antidepressants, psychotherapy or by their association.

\section{The effect of ED treatment on alexithymia}

Overview of studies

Ten out of 15 selected studies examined the effects of specific treatments for ED on alexithymia levels. ${ }^{10,11,13,14,18,19,21-24}$ All studies were based on a longitudinal design; two studies provided for two treatment arms, ${ }^{21,24}$ one study compared 
the efficacy of an experimental intervention with routine treatments, ${ }^{24}$ and two studies included a control group of healthy subjects. ${ }^{10,11}$ The methodology adopted by each study and the treatment protocols used are shown in Table 2. Almost all studies included samples exclusively or predominantly made up of female patients. The diagnostic groups considered in these studies generally consisted of both mixed groups of AN, BN, and EDNOS, , 13,14,23,24 although some studies focused solely on patients with bulimia, ${ }^{10,11} \mathrm{BED},{ }^{21,22} \mathrm{AN},{ }^{19}$ or with BED and EDNOS. ${ }^{18}$ The duration of treatment varied from a minimum of 9.6 weeks $^{11}$ to a maximum of 40 weeks, ${ }^{19}$ with a wide variability between studies. Similarly, treatment protocols displayed a high variability. With regard to treatments, only one of the studies considered included a pharmacological approach. ${ }^{10}$ In all other cases, studies were based on routine group psychotherapy, with individual therapy eventually being associated with other psychological and/or pharmacological treatments; the latter were delivered on a day hospital or inpatient basis. Other studies included only outpatients. Psychotherapeutic approaches varied considerably from one study to another (CBT, psychodynamically oriented therapy, IPT, and PET), and were frequently interassociated. In some cases, the treatment protocol included specific sessions or interventions aimed at improving the recognition, regulation, and/or expression of emotions. ${ }^{13,14,22,24}$ Mostly, studies evaluated the effects of treatments using only total scores obtained at TAS, with the exception of the studies conducted by Ciano et al, ${ }^{21}$ Becker-Stoll and Gerlinghoff, ${ }^{13}$ and Storch et al, ${ }^{24}$ where even TAS dimensions were taken into consideration. Alexithymia levels were invariably measured at the beginning and the end of treatment, with one or more assessments in subsequent follow-ups being provided only in a limited number of studies. ${ }^{19,21,22,24}$ In $60 \%$ of the studies examined, the authors found a significant or quasisignificant reduction of TAS total scores and/or DIF or DDF scores after treatment. ${ }^{11,13,14,18,21,22}$ However, even in cases where alexithymia levels decreased following treatment, the latter frequently remained high, a finding which gave rise to concerns as to the clinical significance of the registered change. ${ }^{11,13}$ When a statistically significant change, in clinical terms, was detected, the magnitude of differences in scores obtained prior to and following treatment was often seemingly low, as is the case in the study conducted by Balestrieri et al. ${ }^{18}$ Four studies found a trend toward a significant improvement in levels of alexithymia following treatment. ${ }^{10,19,23,24}$ Although treatments including specific intervention on emotions were seemingly associated with a greater efficacy on alexithymia, the relevant heterogeneity in methods of treatment and other considerable methodological limitations make it difficult to generalize and compare these results.

\section{Analysis of single studies \\ Single-arm studies}

The first study to analyze the impact of ED treatment on alexithymia was the abovementioned prospective, 10-week study by Schmidt et $\mathrm{al}^{10}$ of 41 patients with bulimia undergoing both psychotherapy and drug treatment (fluvoxamine). After treatment, no significant change in TAS total score was observed, although ED symptoms significantly improved. According to the authors, this suggests that alexithymia cannot be modified by merely improving the core symptoms of ED.

The study by de Groot et $\mathrm{al}^{11}$ evaluated changes to alexithymia in a sample of 31 women with severe $\mathrm{BN}$, compared to a control group of 20 healthy nurses. The treatment involved an intensive 9.6-week program based on group psychotherapy on an outpatient basis, with a focus on nutrition, body image, symptom management, relationships, and family interactions. After treatment, the levels of alexithymia measured by means of TAS-26 were significantly improved, in particular for the group of abstinent subjects (ie, those with no binge/purge episodes), although scores remained significantly higher in comparison to healthy controls. Before treatment, $61.3 \%$ of patients were alexithymic compared to $32.3 \%$ after treatment and $5 \%$ of controls.

Becker-Stoll and Gerlinghoff ${ }^{13}$ examined the impact of a 4-month group therapy program on levels of alexithymia. The study enrolled 47 patients with ED (18 AN, $25 \mathrm{BN}$, four EDNOS). The program included an outpatient phase of motivation (4 weeks), a day hospital treatment phase (4 months) and an outpatient follow-up (4 months). Psychotherapeutic methods included CBT, PET, and IPT. During sessions of group psychotherapy, understanding and expression of emotion through different forms of art therapy was encouraged. A significant improvement of both ED symptoms and alexithymia was registered after treatment, regardless of diagnosis. The most significant improvement was observed for the DIF factor of TAS, with a lower impact, at the limits of statistical significance, on the factor Externally Oriented Thinking, particularly in the bulimic group. Despite the improvement of TAS alexithymia scores, patients prevalently continued to be considered alexithymic on a categorical basis.

Clyne et $\mathrm{al}^{22}$ evaluated the efficacy of an 11-week psychoeducational group program including training on emotion regulation in 11 women with BED. At the end of treatment, not only was effectiveness of treatment on ED symptoms 
demonstrated, but also a significant reduction of levels of alexithymia; for the latter, a further reduction of TAS scores was found at 2-3 months follow-up.

Shiina et $\mathrm{al}^{14}$ evaluated the effectiveness of a 10 -week treatment program combining CBT with assertiveness training and self-esteem reinforcement therapy (CBT combined group) in a group of 25 patients with bulimic symptoms (BN, AN-B/P, EDNOS, and binge eating). During the final stages of treatment, two role play sessions in which patients were required to recognize their emotions and try to express them were added to the program, with the aim of improving alexithymia. Indeed, the program proved to be effective not only for ED, self-esteem, and social functioning, but even for alexithymia, given that TAS-20 total score showed a trend toward a statistically significant improvement from pre- to post-treatment $(P=0.06)$.

Iancu et $\mathrm{al}^{23}$ evaluated the effects of a combined intervention (group and individual psychotherapy, and pharmacological treatment) on 30 young soldiers (three males, 27 females) with ED (ten AN, 15 BN, five EDNOS, diagnosed according to $D S M-I V$ criteria), enrolled in the Israel Defense Forces. The treatment included 6 months of weekly group therapy sessions based on a cognitive behavioral approach combined with a dynamic approach; if necessary, individual psychodynamic psychotherapy or pharmacological treatment was offered. Dietary counseling was strongly suggested, but not necessarily required. Five patients received paroxetine and four patients individual therapy as adjunctive treatments. The level of change of TAS-26 scores obtained after treatment was minimal compared to the improvement in eating symptoms.

In the study by Balestrieri et $\mathrm{al}^{18}$, the authors analyzed the effectiveness of a short-term (10 weeks) outpatient psychoeducational group treatment in patients with BED and EDNOS. Each session included a nutritional intervention, analysis of thoughts and behaviors related to ED, and assertiveness training. After treatment, the patients showed a significant improvement in total TAS, although the clinical significance of this change appears to be negligible (TAS-20 total scores at $\mathrm{t} 0=57 \pm 12 ; 55 \pm 14$ at $\mathrm{t} 1, P=0.035$ ).

Finally, Ohmann et $\mathrm{al}^{19}$ evaluated the effectiveness of a 10-month, multimodal group intervention based on CBT, performed on an outpatient basis; the study also included 29 adolescent girls with AN. The program was made up of nine modules (therapeutic motivation, psychoeducation, individual problem analysis, teaching of problem solving strategies, communication skills, hedonistic training, elements of awareness, body, and schema psychotherapy) and family sessions on a monthly basis. Individual CBT was offered on request. Evaluations were performed at baseline, during treatment (at 3, 6, and 9 months), 1 year after the start of treatment, and 1 year after the end of treatment. Alexithymia proved to be a psychological dimension resistant to change, while body mass index, eating behavior, mood, social anxiety, self-care, and self-efficacy showed significant improvements; however, lack of efficacy of treatments in reducing alexithymia was particularly evident among poor outcome patients.

\section{Two-arm studies}

Ciano et $\mathrm{al}^{21}$ evaluated the effectiveness of two types of group therapy in eleven female patients with BED. Six patients were included on a nonrandomized basis in a group of brief psychoanalytic psychotherapy (14 sessions, 28 weeks), and five patients in a group of PET (ten sessions, 10 weeks). The main purpose of analytical group psychotherapy was to improve self-esteem and, consequently, the control of craving. According to the authors, the homogeneity of the group allowed a "high cohesion and mutual mirroring, promoting awareness of the link between emotions and behavior." PET focused on information about nutrition, communication skills and assertiveness, and nutritional rehabilitation. The treatment provided for role playing and problem solving aimed at changing dysfunctional attitudes regarding eating. Follow-ups were carried out at 6 and 12 months. After treatment, both interventions proved to be effective on ED, but only psychoeducation was associated with an improvement of scores for DIF and DDF factors of TAS-20, during treatment and at each subsequent follow-up, with a significant reduction in scores of only DDF at 6 months follow-up.

Storch et $\mathrm{al}^{24}$ examined the effects of an additional psychoeducational group training aimed at aiding recognition of bodily sensations related to emotion, and improving emotional regulation skills. Nineteen female patients with ED, 15 with AN, and four with BN diagnosed according to $D S M-I V$ criteria were recruited to the study. Evaluations were performed at baseline and 3 and 12 months after treatment. Patients were consecutively assigned to the control group $(n=11)$, receiving standard hospital care, or to the treatment group $(n=8)$, receiving psychoeducation training in addition to the standard hospital care. Four hourly training sessions were delivered for three consecutive Mondays, with a booster session after 8 weeks. Only DIF and DDF subscales of TAS-26 were evaluated, being considered the dimensions with the highest involvement in ED. After treatment, DIF scores were reduced in both groups compared to baseline levels, although the differences detected were 
not statistically significant. At 3 and 12 months follow-up, the addition of psychoeducational training was associated with a significant improvement in the ability to regulate negative affect and with a lower food restriction compared to standard treatment.

\section{Discussion}

Despite the clinical relevance of alexithymia for ED, studies focusing on its therapeutical implications are still limited, being characterized by heterogeneous designs and significant methodological limitations. In the majority of cases, the samples studied are relatively small and made up of patients with different types of ED. The period of observation in many longitudinal studies is too short, with evaluations of alexithymia being carried out largely only at baseline and post-treatment. Criteria adopted to assess clinical improvement are often poor and, in many cases, outcome measures do not take into account the level of clinical change over time. Moreover, the majority of studies does not provide for control or treatment comparison groups. Although TAS is viewed as the most reliable instrument, none of the studies considered in this review include other adjunctive methods of evaluation of alexithymia, and refer to alexithymia as a unitary construct, without taking into consideration its single dimensions. In this regard, to improve the quality of future research, a multidimensional multimethod approach to evaluation of alexithymia has been strongly suggested. ${ }^{6}$ Lastly, published studies frequently fail to give due consideration to confounding factors such as depression.

Overall, data currently available tend to correlate higher levels of alexithymia with a less favorable clinical outcome. Moreover, interventions resulting in a reduction of alexithymic traits seem to be associated with a higher probability of recovery of these patients. As underlined previously, the majority of studies viewed alexithymia as a unitary construct, correlating total scores at TAS with outcome variables. When specific dimensions of the construct were considered, higher levels of DIF emerged as the best predictor of a negative outcome, being mostly negatively correlated with clinical improvement. ${ }^{15}$ Indeed, patients featuring a higher degree of difficulty in identifying emotions at baseline were more often symptomatic at follow-up, and displayed a less favorable clinical improvement. Accordingly, a multidimensional approach that takes into account the influence of the dimensions of alexithymia, would seem to be more informative, allowing the impact of each aspect of the construct on other psychopathological variables to be assessed; however, data currently available to this regard are limited. ${ }^{15}$ The significance of TAS-DIF in terms of predictive power remains, albeit to a lesser extent, even when impact of the depressive component and treatments received is taken into account. ${ }^{15}$ With regard to the effects of specific ED treatments on levels of alexithymia, available data show that alexithymia may be, at least in part, a modifiable trait. Indeed, the majority of studies analyzed reported a somewhat relevant improvement in total TAS score after treatment; moreover, treatments comprising specific intervention on emotions seemed to be characterized by a more pronounced efficacy. ${ }^{13,14,22}$ However, the wide heterogeneity in treatment methods adopted and, in general, the significant methodological limitations of the studies confounds the possibility of generalizing and comparing the emerging results. The majority of studies reporting data on the impact of treatments on alexithymia focus solely on use of TAS total scores. Where single dimensions of the TAS were considered, the most marked change after treatment was registered for DDF and/or DIF, which proved to be more responsive to treatment. ${ }^{13,21,24}$ Based on these results, it has been suggested that some components of alexithymia may remain stable over time, while others may respond to treatment. ${ }^{25}$ It should be emphasized, however, that even in cases in which a reduction of TAS scores was registered after treatment, the overall levels of alexithymia often continued to remain high. ${ }^{11,13}$ Thus, the meaning of these changes in clinical terms is questionable, and highlights the need to identify more precisely which treatments produce a really significant impact on alexithymia. It also remains a matter of debate whether alexithymia should be considered a state or trait variable. ${ }^{9}$ In the study by de Groot, ${ }^{11}$ the partial reversibility of alexithymia was potentially related to several factors, including not only the direct effect of therapy but also the improvement of depressive and ED symptoms, thus demonstrating the complexity of the phenomenon. Thus, changes in alexithymia levels seem to be in some way associated with improvement in eating and/or depressive symptoms. However, some studies, including a study by Schmidt et al, ${ }^{10}$ have reported high levels of alexithymia even after remission of specific symptoms of ED, failing to detect significant changes in TAS, even in the presence of an improvement of ED symptoms; this finding led the authors to conclude that alexithymia should be considered an independent trait, not strictly related to the change in clinical status of ED. Similarly, Iancu et $\mathrm{al}^{23}$ observed a marginal level of change in TAS scores in contrast with a marked improvement of ED symptoms, adding further support to the hypothesis by Schmidt et $\mathrm{al}^{10}$ that alexithymia 
might be an independent psychological dimension, not influenced by post-treatment clinical improvement of ED, unless patients had been offered long-term psychological intervention specifically aimed at ameliorating recognition and expression of emotions. It was also postulated that improvement of ED symptoms may only represent the first, more obvious sign of change, and that a deeper change involving an emotional insight may require longer periods of treatment. ${ }^{23}$ With regard to the influence of depression and, in general, of general distress on alexithymia levels, data are somewhat conflicting. In some studies, levels of alexithymia were found to be closely related to depressive symptoms ${ }^{26,27}$ in other studies, levels of alexithymia were found to be high even after controlling for the depressive component. ${ }^{11,28}$ Overall, the data available indicate prevalently that alexithymia is an independent construct partially linked to depression. ${ }^{9}$ In the study by Speranza et al, ${ }^{20}$ patients with high, stable levels of alexithymia tended to receive more antidepressant treatments compared to nonalexithymic subjects, although depression levels were comparable between the two groups at the time of inclusion in the study. This finding could be explained by a possible wrong attribution by healthcare professionals of higher levels of depression to ED alexithymic patients, and the subsequent prescribing of more antidepressants than needed. Indeed, the use of antidepressants in these patients was related to significant changes only in levels of depression, failing to affect alexithymia levels. In general, in view of the acknowledged association between depression and alexithymia, it is crucial that any studies undertaken should include adequate measurement of depression. Based on evidence present in the literature, a series of findings supporting the hypothesis whereby alexithymia plays a significant role in justifying the outcome of ED: negative influence on clinical expression of the disorders and on response to therapeutic interventions; relationship of alexithymia with other psychopathological dimensions and behaviors; and the effect produced on treatment choices and therapeutic settings. However, a basic question remains to be answered: which components of treatments routinely adopted in ED patients afford the greatest benefits in patients with alexithymia? Data available in literature underline how therapeutic approaches that emphasize the identification, description, and understanding of emotional states appear to be more effective in reducing alexithymia. ${ }^{25}$ At the same time, alexithymic patients seem to prefer and discretely benefit from a therapeutic setting based on group interventions. ${ }^{25}$ Indeed, this context provides patients with the opportunity of observing and modeling their behavior on that of other members capable of describing more effectively their emotional states, consequently receiving feedback on their style of communication, and being encouraged to describe their feelings as others do; in this way, their emotional arousal may be diluted, with a lower risk of somatic expression and of acting merely as passive observers. ${ }^{25}$ Literature findings underline the inappropriateness of assuming that alexithymic patients are largely reluctant to undergo psychotherapy. ${ }^{29}$ Similarly, the widespread opinion that alexithymic patients are not suitable for psychodynamic approaches has very little supporting evidence, as allegedly highly alexithymic subjects may benefit more from psychodynamic therapies based on supportive elements, a strong therapeutic alliance, and a more active role and empathy from the therapist. ${ }^{25}$ In the study by Speranza et al,${ }^{20}$ patients were found to have received different treatments, both qualitatively and quantitatively, in relation to the alexithymia profile. The authors speculated that, given the poorer response of alexithymic patients to traditional therapeutic approaches, the clinician may be induced to consider this type of subject less suited to psychotherapy. ${ }^{20}$ High levels of alexithymia have been shown to evoke negative reactions in therapists, and, in general, negative interpersonal processes; this may elicit countertransference feelings of frustration or boredom, which could hinder a good therapeutic alliance and, consequently, contribute to a negative outcome. $^{29}$

\section{Conclusion}

A pressing need to achieve a better outcome in the treatment of ED underlines the urgency of identifying reliable outcome predictors and personalized care approaches aimed at new treatment targets. Despite the limitations described previously, data currently available in literature support the relevance of a significant impact of alexithymia on outcome of ED treatment. Contemporarily, a moderate impact of ED treatments on alexithymia has also been demonstrated, with a trend for greater efficacy of treatments, including specific interventions on emotions. The fact that alexithymia levels often remain high even when symptoms improve underlines the need for treatments proving more effective on this dimension to be identified. Further research should be carried out using the most appropriate methodological options to provide further insight into the role of alexithymia in ED, focusing in particular on the choice of treatments and identifying treatment protocols better suited for use in ED patients with pronounced alexithymic traits. 


\section{Disclosure}

The authors report no conflicts of interest in this work.

\section{References}

1. Wilson GT, Grilo CM, Vitousek KM. Psychological treatment of eating disorders. Am Psychol. 2007;62(3):199-216.

2. Kass AE, Kolko RP, Wilfley DE. Psychological treatments for eating disorders. Curr Opin Psychiatry. 2013;26(6):549-555.

3. Agras WS, Robinson AH. Forty years of progress in the treatment of the eating disorders. Nord J Psychiatry. 2008;62 Suppl 47:19-24.

4. Bodell LP, Keel PK. Current treatment for anorexia nervosa: efficacy, safety, and adherence. Psychol Res Behav Manag. 2010;3:91-108. Epub October 19, 2010.

5. Sifneos PE. The prevalence of 'alexithymic' characteristics in psychosomatic patients. Psychother Psychosom. 1973;22(2):255-62.

6. Taylor GJ, Bagby RM. New trends in alexithymia research. Psychother Psychosom. 2004;73(2):68-77.

7. Taylor GJ, Ryan D, Bagby RM. Toward the development of a new selfreport alexithymia scale. Psychother Psychosom. 1985;44(4):191-199.

8. Bagby RM, Parker JD, Taylor GJ. The twenty-item Toronto Alexithymia Scale - I. Item selection and cross-validation of the factor structure. J Psychosom Res. 1994;38(1):23-32.

9. Nowakowski ME, McFarlane T, Cassin S. Alexithymia and eating disorders: a critical review of the literature. J Eat Disord. 2013;1:21.

10. Schmidt U, Jiwany A, Treasure J. A controlled study of alexithymia in eating disorders. Compr Psychiatry. 1993;34(1):54-58.

11. de Groot JM, Rodin G, Olmsted MP. Alexithymia, depression, and treatment outcome in bulimia nervosa. Compr Psychiatry. 1995;36(1): 53-60.

12. Beales DL, Dolton R. Eating disordered patients: personality, alexithymia, and implications for primary care. $\mathrm{Br} J$ Gen Pract. 2000;50(450):21-26.

13. Becker-Stoll F, Gerlinghoff M. The impact of a four-month day treatment programme on alexithymia in eating disorders. Eur Eating Disord Rev. 2004;12(3):159-163.

14. Shiina A, Nakazato M, Mitsumori M, et al. An open trial of outpatient group therapy for bulimic disorders: combination program of cognitive behavioral therapy with assertive training and self-esteem enhancement. Psychiatry Clin Neurosci. 2005;59(6):690-696.

15. Speranza M, Loas G, Wallier J, Corcos M. Predictive value of alexithymia in patients with eating disorders: a 3-year prospective study. J Psychosom Res. 2007;63(4):365-371.

16. Leweke F, Bausch S, Leichsenring F, Walter B, Stingl M. Alexithymia as a predictor of outcome of psychodynamically oriented inpatient treatment. Psychother Res. 2009;19(3):323-331.
17. Tchanturia K, Davies H, Harrison A, Fox JR, Treasure J, Schmidt U. Altered social hedonic processing in eating disorders. Int J Eat Disord. 2012;45(8):962-969.

18. Balestrieri M, Isola M, Baiano M, Ciano R. Psychoeducation in Binge Eating Disorder and EDNOS: a pilot study on the efficacy of a 10-week and a 1-year continuation treatment. Eat Weight Disord. 2013;18(1):45-51.

19. Ohmann S, Popow C, Wurzer M, KarwautzA, Sackl-Pammer P, Schuch B. Emotional aspects of anorexia nervosa: results of prospective naturalistic cognitive behavioral group therapy. Neuropsychiatr. 2013;27(3): $119-128$.

20. Speranza M, Loas G, Guilbaud O, Corcos M. Are treatment options related to alexithymia in eating disorders? Results from a three-year naturalistic longitudinal study. Biomed Pharmacother. 2011;65(8): $585-589$.

21. Ciano R, Rocco PL, Angarano A, Biasin E, Balestrieri M. Group-analytic and psychoeducational therapies for binge-eating disorder: an exploratory study of efficacy and persistence of effects. Psychother Res. 2002;12(2):231-239.

22. Clyne C, Latner JD, Gleaves DH, Blampied NM. Treatment of emotional dysregulation in full syndrome and subthreshold binge eating disorder. Eat Disord. 2010;18(5):408-424.

23. Iancu I, Cohen E, Yehuda YB, Kotler M. Treatment of eating disorders improves eating symptoms but not alexithymia and dissociation proneness. Compr Psychiatry. 2006;47(3):189-193.

24. Storch M, Keller F, Weber J, Spindler A, Milos G. Psychoeducation in affect regulation for patients with eating disorders: a randomized controlled feasibility study. Am J Psychother. 2011;65(1):81-93.

25. Cameron K, Ogrodniczuk J, Hadjipavlou G. Changes in alexithymia following psychological intervention: a review. Harv Rev Psychiatry. 2014;22(3):162-178.

26. Eizaguirre AE, de Cabezon AOS, de Alda IO, Olariaga LJ, Juaniz M. Alexithymia and its relationships with anxiety and depression in eating disorders. Pers Indiv Differ. 2004;36:321-331.

27. Parling T, Mortazavi M, Ghaderi A. Alexithymia and emotional awareness in anorexia nervosa: time for a shift in the measurement of the concept? Eat Behav. 2010;11(4):205-210.

28. Sexton MC, Sunday SR, Hurt S, Halmi KA. The relationship between alexithymia, depression, and axis II psychopathology in eating disorder inpatients. Int J Eat Disord. 1998;23(3):277-286.

29. Ogrodniczuk JS, Piper WE, Joyce AS. Effect of alexithymia on the process and outcome of psychotherapy: a programmatic review. Psychiatry Res. 2011;190(1):43-48.
Psychology Research and Behavior Management

\section{Publish your work in this journal}

Psychology Research and Behavior Management is an international, peerreviewed, open access journal focusing on the science of psychology and its application in behavior management to develop improved outcomes in the clinical, educational, sports and business arenas. Specific topics covered include: Neuroscience, memory \& decision making; Behavior

\section{Dovepress}

modification \& management; Clinical applications; Business \& sports performance management; Social and developmental studies; Animal studies. The manuscript management system is completely online and includes a quick and fair peer-review system. Visit http://www.dovepress. com/testimonials.php to read real quotes from published authors. 